\title{
Self-Positioning System and Algorithm in Wireless Sensor Networks
}

\author{
Liu Ting ${ }^{1, \text { a }}$, Shuai Zhijun ${ }^{2}$ \\ ${ }^{1}$ Jiangxi Modern Polytechnic College, Nanchang 330095, China \\ àiutingnc@126.com
}

Keywords: wireless sensor network, self-localization, positioning

\begin{abstract}
Wireless Sensor Networks, a novel technology about acquiring and processing information, have been proposed for a multitude of diverse applications. The problem of self-localization, that is, determining where a given node is physically or relatively located in the networks, is a challenging one, and yet extremely crucial for many applications. In this paper, the evaluation criterion of the performance and the taxonomy for wireless sensor networks self-localization systems and algorithms are described, the principles and characteristics of recent representative localization approaches are discussed and presented, and the directions of research in this area are introduced.
\end{abstract}

\section{Introduction}

MEMS, wireless communication and digital electronic technology gave birth to the wireless sensor network (wireless sensor network, referred to as WSN). Through the deployment of a large number of sensor nodes to the target area, WSN will change the way we and the objective world. Interactive exploration from the military application, target tracking, environmental monitoring to the space, the future application of WSN will be beyond our imagination.

For most applications, do not know the position sensor. sensor node sensing data are of no significance to clear their position to a detailed description of some events occur in what position or area, realize the external target positioning and tracking. on the other hand, to understand the location information of sensor nodes can also improve the routing efficiency, provide namespace for the network to report the deployment of network coverage quality, achieve load balance and network topology network self-configuration. and artificial deployment and for all network nodes to install the GPS receiver is limited by cost, power consumption, scalability and other issues, and even in some cases may not can not be achieved, so we must adopt a mechanism and algorithms of WSN localization.

\section{Performance Evaluation of Wireless Sensor Network Self Positioning System and Algorithm}

The performance of WSN self-localization systems and algorithms directly affect its availability, how to evaluate them is a problem for further study.

The qualitative discussion of several evaluation criteria. Positioning accuracy. The evaluation index is the primary positioning technology positioning accuracy, general error value and the proportion of nodes in the wireless range, for example. The positioning accuracy is $20 \%$. Positioning error is equivalent to the node of the wireless range are also part of the two-dimensional positioning system network deployment area is divided into grid, its location accuracy is the size of the grid, such as Microsoft RADAR, Wireless Corporation Radio Camera.

Scale. Therefore, one of the important indexes of the anchor node density but also evaluate the positioning system and the performance of the algorithm. Node density. In WSN, the node density increases not only means to increase the network deployment costs, but because the nodes Blocking communication conflicts bring limited bandwidth. Due to the environment, energy consumption and other reasons, physical maintenance or replacement of the sensor nodes or the use of other high-precision measurement instruments are often very difficult or not feasible. Therefore, the 
hardware and software localization systems and algorithms must have fault tolerance and strong adaptability, can influence through automatic adjustment or reconstruction correct the mistakes and adapt to the environment, reduce the error, to improve the positioning accuracy.

Power. Power is the design and Realization of WSN one of the most influential factors. Because the energy of the sensor node Battery Co., therefore, under the precondition of ensuring accuracy, computation, positioning is closely related with the power required for communication overhead, the storage overhead, the time complexity is a set of key indicators.. price positioning system or algorithm from several different aspects.

To evaluate the time cost of including a system. Time, time, time allocation to locate the required quantity and space. The price includes a positioning system or algorithm required infrastructure and network node hardware size. The total cost of capital cost including infrastructure, node equipment to achieve a positioning system or algorithm.

\section{The Classification of the Self Positioning System and Algorithm of Wireless Sensor Networks}

The positioning system can provide positioning results of two types: physical position and symbolic position. For example, a node is located at 47 degrees 39 '17 "N, 122 degrees 18' 23" W is the physical location; and a node in the building room 123 is the symbol position. Under certain conditions, the physical location and symbols positioning can transform each other.

Compared with the physical location, symbol location is more suitable for some special applications, for example, in the installation of intelligent building wireless sensor network in fireworks, managers are more concerned about whether a room or area of fire signal, rather than the fire occurred in the longitude and latitude. Most of positioning system and algorithm provide the physical location services, typical systems and algorithms are Active symbol location Badge, Microsoft Easy Living, Cricket MIT positioning system can be configured according to two different forms of positioning. Absolute positioning and physical mapping of similar positioning results is coordinates of a standard, such as longitude and latitude.

While the relative positioning is usually to some nodes in the network as the reference, the relative coordinate system establishment of the entire network. Absolute positioning can provide only a namespace for the network, less affected by the node mobility, there is a wider application field. But the study found that can realize some routing protocols based on relative positioning, especially geographical location based routing (geo-routing), and the relative positioning does not need the anchor node. Most localization systems and algorithms can achieve absolute positioning service, relative localization algorithm and typical system of SPA (self-positioning algorithm LPS (local), positioning system), Spoton, and MDS-MAP localization algorithm in two position respectively according to the network configuration.

\section{Tightly Coupled Versus Loosely Coupled}

The so-called tightly coupled positioning system refers to the anchor node not only be carefully deployed in a fixed position, and through the wired medium connected to the central controller; and node loose type positioning system using distributed wireless coordination mode without a central controller. Typical tightly coupled positioning system including Active Bat and Active system Badge AT\&T HiBall Tracker,. Their characteristics are applicable to indoor environment, real-time and accuracy is high and the coordination problem of time synchronization between the anchor node and easy to solve. But this deployment strategy limits the scalability of the system, the cost is large, can not be used in outdoor environment is not feasible. Wiring in recent years, put forward many localization systems and algorithms, such as Cricket, Ahlos etc. are loosely coupled solutions. Their accuracy at the expense of tightly coupled system the cost obtained the deployment flexibility, rely on inter node coordination and information exchange to achieve positioning. In loosely coupled systems, because the network using adhoc deployment, there is no direct coordination between nodes, so nodes will channel competition and interaction. To solve this problem, Cambridge Mike 
Hazas in the literature in the proposed Wide band Spread Spectrum Technology to solve the problems of noise interference and multiple access zone. This classification method based on and infrastructure and without infrastructure (infrastructure-based versus infrastructure-free) classification method is similar to that, the difference is that the latter is based on the entire system in addition to the sensor nodes are outside other facilities as the standard.

\section{Centralized Computation Versus Distributed Computation}

Centralized computing refers to the required information is transmitted to a central node (e.g., a server), the calculation method and node location where; distributed computing refers to rely on information exchange among nodes and coordination, positioning by the node to calculate. The advantages of centralized computing is the overall planning from a global perspective the amount of calculation and storage, almost no restrictions, you can get a relatively accurate position estimation. Its disadvantages include with the position of the center node closer because of communication overhead and prematurely consumed power, cause the interruption of the whole network and center node information exchange, real-time positioning. centralized localization algorithms including convex planning (convex optimization), localization algorithm according to application requirements by two different calculation models.

\section{Typical Self Positioning System and Algorithm.}

Relative localization algorithm SPA. It selects a set of nodes in the network with the maximum density as the global coordinate system to establish a network of reference points (called the location reference group), and in which a node is selected as the maximum degree of the origin of the coordinate system. According to the distance between nodes results in each node the establishment of local coordinate system, through the exchange of information between nodes and coordination on the reference point for reference by coordinate transformation (translation and rotation) to establish the global coordinate system. Because the establishment and transformation of all nodes are required to participate in the coordinate calculation, so the quantity of communication overhead and node SPA almost exponentially over. Aiming at the shortcoming of SPA. The Jin J Lear Lee Institute (Rensselaer Polytechnic Institute) Rajagopal Iyengar. Proposed based on clustering (clustering-based) algorithm: network deployment, each all nodes start running a random timer. If compared with the neighbor node, the node I is the earliest timer expires, I becomes a master node (Master), and broadcast a message to the neighbor node, all received the message of the neighbor node to end its timer and into a slave node (slave), these nodes form a domain. Then based on these main node to the local coordinate system origin using similar methods and SPA of each domain. Then the local coordinate system of the master node ID is smaller as a reference, the neighboring domain by coordinate transformation, and gradually establish a global coordinate system. Because the traffic is increasing the number of domains, so the number of nodes and network communication overhead of the algorithm is linear, more suitable for large-scale network deployment. Obviously, relative localization algorithm is the biggest problem when the reference point moved or failure, The whole network must be relocated.

\section{Convex Programming Location Algorithm}

Doherty of the University of California at Berkeley and others will be a point-to-point communication between nodes as geometric constraint nodes, the whole network is modeled as a convex set, thus the node localization problem is transformed into a convex optimization problem, and then use the marking method of semidefinite programming and linear programming to get a global optimal solution to determine the node location at the same time. A method to calculate the unknown node rectangle possible is provided. As shown, according to the communication between the unknown nodes and anchor nodes and nodes in the wireless connection range, calculate the unknown nodes may exist in the region (shaded part), and the corresponding rectangular area, and 
then to rectangular centroid as the location of the unknown node.

\section{Summary}

According to the above discussion, each kind of systems and algorithms have their own characteristics and scope of application, no one is absolutely the best. On the whole, in recent years some of the iterative refinement algorithm, such as Cooperative ranging, Two-phase positioning, n-hop multilateration primitive more, giving full play to the WSN the characteristics, namely the use of collaborative work between nodes to achieve a single node cannot complete the task.

They are in the initial stage focuses on the rough estimate obtained the position of the nodes, and in the refinement stage according to the precision threshold loop preset by the user; even according to the application requirements, the refinement stage as an optional stage.

These algorithms not only improve the positioning precision, but also give the user more freedom in, has gradually formed a new category of. But we should also see that the positioning system and the existing algorithms are as follows some of the problems: unknown nodes should be directly adjacent to the anchor node and anchor node density is too high, such as Cricket, Cooperative ranging. Accuracy depends on the network conditions.

By cyclic refinement process and improve the positioning accuracy of the ranging error suppression. Although the refinement process can significantly affect the circulation to reduce the ranging error, but not only has high communication and computation costs, but also because of the number of cycles and increase can not be estimated with the algorithm of uncertainty. For example, Cooperative ranging Two-phase, positioning, n-hop 866 Journal of Software Journal of software 2005,16 (5) multilateration primitive. Algorithm converges slowly. For example, Generic Localized Algorithm algorithm in each cycle, only one adjacent node in the positioning can be realized and upgraded as anchor nodes.

Simulation shows that the positioning accuracy of many algorithms and systems have greatly improved space. In a word, study the localization problem in wireless sensor networks is still one of the key technologies of WSN, and it is also one of the key points.

\section{Expectation}

In the past 10 years, research progress of WSN positioning itself is very gratifying, made great achievements. Especially in twenty-first Century, WSN of positioning itself has many innovative solutions and ideas.

However, research in this area that is still in a starting stage on the whole the previous research work is put forward, more and more problems need to be solved in this field. We believe that in addition to research on the positioning algorithm itself, including the focus of possible research directions: to model and quantify the localization algorithm performance evaluation; to achieve the establishment of standard simulation technology and simulation system to simulate the positioning algorithm; low cost itself location is composed of tens of thousands of nodes in large or super large scale network (time, power and price) and high precision; in the mobile network environment with self-positioning adjustment algorithm The realization of the law.

\section{References}

[1] Ren FY, Huang HN, Lin C. Wireless sensor networks. Journal of Software, 2003, 14(2):1148-1157 (in Chinese with English abstract).

[2] Bahl P, Padmanabhan VN. RADAR: An in-building RF-based user location and tracking system. In: Proc. of the IEEE INFOCOM 2000. Vol.2, Tel Aviv: IEEE Computer and Communications Societies, 2000.

[3] Beutel J. Geolocation in a PicoRadio environment [MS. Thesis]. Berkeley: UC Berkeley, 1999.

[4] Bulusu N, Heidemann J, Estrin D. GPS-Less low cost outdoor localization for very small 
devices. IEEE Personal Communications, 2000, 7(5):28-34.

[5] Rabacy JJ, Ammer MJ, da Silva Jr. JL, Patel D, Roundy S. Picorodio supports ad hoc ultra-low power wireless networking. Computer, 2000, 33(7):42-48.

[6] Savarese C, Rabaey JM, Beutel J. Locationing in distributed ad-hoc wireless sensor network. In: the 2001 IEEE Intl Conf. on Acoustics, Speech, and Signal. Vol.4, Salt Lake: IEEE Signal Processing Society, 2001.

[7] Capkun S, Hamdi M, Hubaux J-P. GPS-Free positioning in mobile ad-hoc networks. Cluster Computing, 2002, 5(2):157167. 